\title{
Time Series Prediction Based on Machine Learning
}

\author{
Q.Y. Jiang \\ College of Computer Science and Information Technology \\ Central South University of Forestry and Technology \\ Changsha, China
}

\begin{abstract}
Time series is an important temporal data object types, for time series prediction has great significance and wide application. This paper studies the key technologies for predicting the time series of machine learning, mainly in the following three points: (1) proposed a two-class nuclear space feature selection frame (2), a fast multi-core-based distance learning method (3) the prediction of time series based on key technologies applied to machine learning model among business intelligence .
\end{abstract}

Keywords-time series forecasting; machine learning; feature extraction

\section{INTRODUCTION}

In chronological order of arrival data, have appeared in many fields, such as finance, physics, biology, medicine and meteorology. Time series is an important temporal data object types, and they are easy to get from the financial sector and scientific applications, machine learning is through comprehensive statistical data to build the model can well describe the distribution of data. Through machine learning, based on the distribution of the data based on a variety of practical activities to law after learning guide. Using machine learning methods for time series forecasting, research has become a point of concern. However, due to the rapid growth of the current time series, large-scale time series data is more widespread, so that the time series prediction based on machine learning is facing a series of major new challenges and deal with the problem quickly, especially high-dimensional data. This paper studies the problem of rapid processing of this critical high-dimensional data.

\section{NUCLEAR SPACE TWO CLASSIFICATION FEATURE SELECTION FRAMEWORK}

This paper presents a classification in the nuclear space, two feature selection framework that can be used either in a supervised feature selection can also be used for semi-supervised feature selection. This is a new feature selection perspective, it makes feature selection problem can be the best of any of the current two-class feature selection methods to solve. Feature selection framework taking into account the impact of individual characteristics and combinations of features to predict or classify the results. For classification problems, the first of the original features projected onto a special kernel space, the paper called the $\mathrm{K}$ space, which is a step in order to better reflect the role of each dimension of features distinguish different categories of data.

Thus, K space may be configured to

$$
\left\{\left(\mathrm{z}_{\mathrm{x}}, \mathrm{x}^{\prime}, \mathrm{t}_{\mathrm{y}}, \mathrm{y}^{\prime}\right) \mid(\mathrm{x}, \mathrm{y}),\left(\mathrm{x}^{\prime}, \mathrm{y}^{\prime}\right) \quad \mathrm{P} \times \mathrm{P}\right\} \quad \mathrm{R}^{\mathrm{m}} \times\{ \pm 1\} \text {, in }
$$

$$
\begin{gathered}
\mathrm{z}_{\mathrm{x}}, \mathrm{x}^{\prime}=\left(\mathrm{K}\left(\mathrm{f}_{\mathrm{x}} 1{ }^{\prime} \mathrm{f}_{\mathrm{x} 1}{ }^{\prime}\right), \mathrm{K}\left(\mathrm{f}_{\mathrm{x}} 2 \mathrm{f}_{\mathrm{x}}{ }^{\prime}\right), \ldots \ldots, \mathrm{K}\left(\mathrm{f}_{\mathrm{x}} \mathrm{m}^{\prime} \mathrm{f}_{\mathrm{x}}{ }^{\prime} \mathrm{m}\right)\right) \\
\text { tyy }^{\prime}=2 \cdot 1\left\{\mathrm{y}=\mathrm{y}^{\prime}\right\}-1
\end{gathered}
$$

This paper proposed two nuclear space classification feature selection framework is based on this special nuclear space. The framework will be to find a "good" features a combination of a transformation by solving the resulting binary classification problem. Theoretical and experimental results show that the proposed method in a supervised and semi-supervised feature selection have very good performance. Further, since there is no data to be distributed to the structural characteristics of the selected any assumptions, the method for the distribution of different data structures are robust.

\section{FAST MULTICORE LEARNING}

Multiple Kernel Learning is a technology that allows classifier and predictor of learning from multi-core to a machine learning method information. Multiple Kernel Learning goal is to learn from a given set of base through a combination of nuclear and nuclear-based construct a new nuclear, making this new nuclear issue can better adapt to the current process. This paper presents a new method based on multi-core ELM, called Distance Based Multiple Kernel Extreme LearningMachine, DBMK-ELM. The two-step method is a fast multi-core learning methods, and can be applied to both classification and regression problems. In the first step, DBMK-ELM first coefficient based on training samples by combining pre-generated based nuclear matrix to find the base core. It is first projected onto the original base nuclear space and a new training samples based on the distance of the original construction of the new training samples in the new space, and then it will be multi-core learning problem into a regression problem and solved by the least squares method Finally, it is based on a combination of learning to base nuclear factor construct new nuclear. The second step, DBMK-ELM will be the first step in learning to new nuclear applied to have the following advantages can be based nuclear ELM among the experimental results show that the proposed method DBMK-ELM: (1) DBML-ELM compared with the traditional method of learning has a fast multi-core training speed; (2) DBMK-ELM from the use of multiple sources of data points and the accuracy of classification and regression method is superior to the previous multi-core learning; (3) DBMK-ELM on the classification and return to the more traditional problem-based nuclear ELM has better robustness and higher accuracy.

\section{STOCK TRADING DECISIONS RECOMMENDATION SYSTEM}

The earlier study of the key technologies based on time series forecasting model being applied to business intelligence 
to build a stock trading system policy recommendations. Based on time-series forecasting stock trading decisions recommendation system architecture consists of the following components: The first part is the data pre-processing section, which comprises extracting feature value data, the key features of each of the feature selection and the calculation of correlation and in accordance with the gray values of weighted feature. The second part is based on the stock price time series prediction part of machine learning, in this section is mainly used DBMK-ELM to learn the stock market historical data mode, and the upper and lower boundaries of the box the next time the shock phase to predict. The last part is the part of the trading strategy, in part, based on the predicted value of the basis for analysis to determine the shock box model based on trading strategies, and ultimately to make trading decisions.

The system combines two nuclear space classification feature selection methods, the third fast multicore learning methods and transaction boundary model, making the system in stock market trading can give a reasonable, reliable, high-yield, low-risk policy recommendations, and has high speed train, able to meet the actual needs of the stock market. Note that for certain stocks yield obtained in this system is much higher than the average yield of the system obtained in the future work will focus on and how to use clustering and semi-supervised learning techniques selected for this system has the potential transaction stock, so the yield is further improved.

\section{SUMMARY}

As an important temporal data type, time series widespread in the real world, with its far-reaching significance Prediction and Research, and machine learning methods just can dig out potential patterns and rules from massive data. From this point of view, the machine learning method is applied to predict the time series into becoming an effective way to grasp the opportunity to overcome the challenges. First, how to choose the key features from high-dimensional data in high-dimensional data not only affects the speed of machine learning, but also may contain redundant information and learning goals unrelated to the high-dimensional features, even the noise contains errors information. This information will lead to a serious decline in the accuracy of machine learning, thereby affecting the accuracy of the time-series forecasting, so vital in forecasting feature selection based on machine learning time. Then the face of large-scale high-precision data quickly learning problems, due to the growing time series over time, the amount of data within some time sequence for each time unit growth is still very large, which requires machine learning at the same time guarantee the accuracy of learning also ensure that the learning speed to keep up with the growth rate of the time series.

\section{ACKNOWLEDGEMENTS}

The research work was supported by Central South University of Forestry and Technology Youth Science Foundation project under Grant No. QJ2011015B.
[1] Yu L,Liu H. Feature selection for high-dimensional data: A fast correlation-based filter solution [C]. In Proceedings of the International Conference on Machine Learning. 2003: 856-863.

[2] Rogati M, Yang Y. High-performing feature selection for text classification [C]. In Proceedings of the eleventh international conference on information and knowledge management. 2002: 659-661.

[3] He X, Cai D, Niyogi P. Laplacian score for feature selection [C]. In Advances in neural information processing systems. 2005: 507-514.

[4] Cotter S F, Kreutz-Delgado K, Rao B D. Backward sequential elimination for sparse vector subset selection [J]. Signal Processing. 2001, 81 (9): 1849-1864.

[5] Colak S, Isik C. Feature subset selection for blood pressure classification using orthogonal forward selection [C]. In IEEE Annual Proceedings of Bioengineering Conference. 2003: 122-123.

[6] Yang J, Honavar V. Feature subset selection using a genetic algorithm [M] // Yang J, Honavar V. Feature extraction, construction and selection.Springer, 1998: 1998: 117-136.

[7] Wang X, Yang J, Teng X, et al. Feature selection based on rough sets and particle swarm optimization [J]. Pattern Recognition Letters. 2007 , 28 (4): 459-471.

[8] Nie F, Huang H, Cai X, et al. Efficient and robust feature selection via joint $\ell 2,1$ norms minimization [J]. Advancess in Neural Information Processing Systems.2010: 1813-1821.

[9] Sun Y, Todorovic S, Goodison S. Local-learning-based feature selection for high-dimensional data analysis [J]. IEEE transactions on pattern analysis and machine intelligence. 2010, 32 (9): 1610-1626.

[10] Peng H, Long F, Ding C. Feature selection based on mutual information criteria of max-dependency, max-relevance, and min-redundancy [J] IEEE transactions on pattern analysis and machine intelligence. 2005, 27 (8): 1226-1238.

\section{REFERENCES}

